\title{
Estrategias de afrontamiento e inadaptación en niños y adolescentes
}

\author{
Francisco M. Morales y $\mathrm{M}^{\mathrm{a}}$ Victoria Trianes \\ Universidad de Málaga (España)
}

\begin{abstract}
El objetivo general del presente trabajo es estudiar la relación entre el empleo de estrategias de afrontamiento, ajuste psicológico y manifestación de comportamientos desadaptados. El enfoque adoptado es situacional, considerando la estrategia en relación con la situación particular, en concreto, se han evaluado las estrategias de afrontamiento del estrés utilizadas por escolares andaluces de educación primaria en relación con cuatro tipos de estresores: escolar, familiar, relaciones con los iguales y salud. Participaron 402 niños entre 9 y 12 años. Los instrumentos utilizados fueron: el IIEC (Inventario Infantil de Estresores Cotidianos), el ESAN (Estrategias situacionales de afrontamiento en niños y en niñas) y el BASC (Sistema de Evaluación de la conducta en niños y adolescentes). Los resultados demuestran un uso diferencial de estrategias de afrontamiento según el problema o situación planteada. El análisis de las estrategias de afrontamiento revela además que el empleo de estrategias de afrontamiento de aproximación a los problemas se asocia a un mayor grado de ajuste y adaptación mientras que un mayor empleo de estrategias de tipo evitativo se asocia con menos ajuste y comportamientos más desadaptativos.
\end{abstract}

Palabras clave: Estrategias de afrontamiento, inadaptación/psicopatología, estrés infantil.

Coping strategies and maladjustment in children and adolescents. The aim of the present study is to analyze the relationship between used coping strategies, psychological adjustment and behavioral problems. The approach adopted to understand stress coping provides a context for each situation or problem, in order to facilitate a more precise understanding of coping strategies used by students of elementary education, and in relation to four types of stressors: school, family, peer interaction and health. The participants were 402 school children from 9 to 12 years old who belonged to Primary Educational levels. The instruments used were: the IIEC (Children`s Daily Stress Inventory), the ESAN (Situational Coping Strategies Inventory in Children), and the BASC (Behaviour Assessment System for Children and Adolescents). The results demostrate that children and adolescents use different coping strategies depending on the situation or problem. The analysis of coping strategies revealed that the coping approach is associated with better psychological adjustment and good outcomes regarding adaptation, while the use of avoidance strategies is related to maladjustment and behavioral problems.

Key words: Coping strategies, maladjustment/psychopathology, childhood stress.

Correspondencia: Francisco Manuel Morales Rodríguez. Universidad de Málaga. Departamento de Psicología Evolutiva y de la Educación. Facultad de Psicología. Campus de Teatinos, s/n. C.P. 29071. Málaga (España). E-mail: framorrod@uma.es 
Las estrategias de afrontamiento empleadas se relacionan con la adaptación y salud mental de niños y adolescentes, ya que el empleo diferencial de estrategias arroja diferentes resultados de adaptación y salud mental. Así, numerosos autores encuentran una relación entre las estrategias empleadas, las situaciones estresantes y una predicción de psicopatología e inadaptación o por el contrario, de salud mental, en niños (Cabanach, Valle, Rodríguez, Pineiro y González, 2010; Compas, Orosan y Grant, 1993; Lapointe y Marcotte, 2000; Seiffge-Krenke, 2000). De hecho, algunos de estos estudios encuentran que el empleo de estrategias de afrontamiento productivas y eficaces ante problemas de convivencia escolar y de deterioro de las relaciones interpersonales, frecuente en la actualidad en los centros educativos, se asocia a resultados más favorables de adaptación socioemocional.

Una investigación clásica centrada en el afrontamiento de niños y adolescentes ante eventos cotidianos estresantes de naturaleza académica e interpersonal (Compas, Malcarne y Fondacaro, 1988) encontró que estrategias de solución activa del problema correlacionaron negativamente con problemas conductuales, mientras que el empleo de estrategias de tipo emocional correlacionó positivamente con problemas conductuales.

En el ámbito de las relaciones con los iguales y ante problemas de disciplina y de convivencia escolar, Griffith, Dubow e Ippolito (2000) han encontrado que, ante estresores en las relaciones interpersonales con iguales, las estrategias de afrontamiento de aproximación/acercamiento al problema así como las búsqueda de apoyo social y una mayor competencia social se asocian a un mejor ajuste en niños y niñas, mientras que las estrategias centradas en la evitación del problema se asocian a un mayor desajuste, concretamente correlacionan positivamente con síntomas emocionales, depresión y ansiedad. Asimismo, otros estudios (Vendette y Marcotte, 2000) encuentran relaciones entre estrategias de afrontamiento de tipo evitativo y trastornos específicos como depresión y ansiedad. No obstante, dichos estudios son complejos y en un número cada vez mayor de investigaciones (Grant et al., 2006; Halama y Bakosova, 2009) se insiste en que todas estas estrategias de afrontamiento pueden ser adaptativas dependiendo del tipo de situación estresante.

Para concluir, conviene destacar que las conductas de afrontamiento han de considerarse como fruto de un proceso que depende de la evaluación que el sujeto realiza de las demandas de una situación o problema concreto. Así, las estrategias de afrontamiento suscitadas ante un problema dado pueden diferir mucho de las empleadas en otras situaciones distintas. Se argumenta la necesidad de intervenciones psicoeducativas ante este tipo estresores que promuevan en el alumnado el desarrollo de estrategias de afrontamiento efectivas. Saber afrontar de forma productiva y desde una perspectiva sistémica todos estos problemas (familia, salud, escuela e iguales) se asocia a 
resultados favorables de adaptación socioemocional y escolar contribuyendo a la mejora del proceso educativo y de la convivencia escolar.

En el presente estudio se analiza cómo el empleo de unas u otras estrategias de afrontamiento ante problemas con los padres, salud, con las notas y con los iguales se asocia con mayor o menor grado de ajuste y adaptación. Así pues, el objetivo general del presente trabajo es valorar el tipo de estrategias de afrontamiento ante distintos tipos de situaciones o problemas utilizadas por escolares andaluces, analizando cómo el empleo de unas u otras estrategias de afrontamiento (más o menos productivas) se asocia con medidas más o menos globales del ajuste psicológico.

\section{METODO}

\section{Participantes}

Los participantes han sido 402 sujetos, 190 niñas (47.26\%) y 212 niños (52.74\%) con edades comprendidas entre los 9 y 12 años, pertenecientes a dos colegios públicos y dos colegios concertados de Málaga, con una media de edad de 10.49 años $(D T=1.00)$. Se trabajó con una muestra incidental no probabilística.

\section{Instrumentos}

Inventario Infantil de Estresores Cotidianos (IIEC, Trianes, Blanca, Fernández, Escobar, Maldonado y Muñoz, 2009): se trata de un inventario diseñado por el Grupo de Investigación HUM-378 con la finalidad de realizar un estudio epidemiológico sobre el estrés infantil cotidiano y otras variables emocionales en población de 9 a 12 años. Consta de 26 ítems dicotómicos a cumplimentar por cada alumno/a y permite evaluar el estrés percibido por acontecimientos cotidianos, con cuestiones referidas a tres campos: salud (12 ítems), escuela e iguales ( 7 ítems) y familia (7 ítems). Permite obtener una puntuación global por autoinforme y presenta adecuadas propiedades psicométricas. Concretamente, en la presente muestra se obtiene una consistencia interna de $\alpha=.81$ para la puntuación total del IIEC, siendo .67 para la dimensión salud, .68 para la de escuela e iguales y .64 para la de familia.

Estrategias situacionales de afrontamiento en niños y en niñas (ESAN, Morales, Trianes, Miranda, Fernández y Escobar, 2008): este instrumento autoinformado ha sido elaborado por el grupo de investigación HUM/0378 del PAI andaluz y chequea estrategias de afrontamiento suscitadas ante cuatro problemas, relacionados con el contexto familiar, la salud, las tareas escolares, y las relaciones sociales. Estas estrategias fueron obtenidas de forma empírica ya que, en un estudio previo, con una muestra de 966 sujetos, las respuestas emitidas ante problemas abiertos fueron categorizadas por dos observadores obteniéndose una correlación media adecuada $(r=.67)$ y diez estrategias de afrontamiento, constituyendo los diez ítems que se anidan en cada uno de los cuatro problemas hipotéticos. La versión final del ESAN consta de 40 ítems, repartidos 
equitativamente en 4 situaciones problema, con un formato de respuesta en 5 escalones. La respuesta se refiere a los últimos 12 meses. Existe, para cada problema, la posibilidad de emitir una respuesta abierta describiendo una estrategia que no se encuentra entre las ofrecidas.

Sistema de Evaluación de la conducta en niños y adolescentes (BASC, Reynolds y Kamphaus, 1992): en este trabajo se ha utilizado la versión española de González, Fernández, Pérez y Santamaría (2004). Concretamente se ha empleado un componente del BASC: el Autoinforme de Personalidad (S2) para niños y niñas con edades entre los 8 y 12 años. Es un inventario formado por 146 enunciados que han de ser contestados como verdadero o falso. Consta de doce escalas que están agrupadas en ocho escalas clínicas y cuatro escalas adaptativas. El BASC arroja cuatro escalas globales o índices compuestos que constituyen variables que se vienen empleando de forma regular en la investigación sobre psicopatología e inadaptación (Keane y Calkins, 2004). Las citadas escalas globales, que se han empleado en el presente estudio, son: desajuste escolar, desajuste clínico, adaptación personal y síntomas emocionales. En la presente muestra la consistencia interna de cada una de las escalas es de $\alpha=.85$ para desajuste escolar, .90 para desajuste clínico, .84 para adaptación personal y .93 para síntomas emocionales.

\section{Procedimiento}

Se contactó con los distintos centros de Educación Primaria informándoles por escrito de los objetivos del proyecto, al mismo tiempo que se solicitó a los padres su autorización y conformidad en la investigación, proporcionándoles las correspondientes instrucciones y notas informativas. Para la administración de las diversas pruebas se informó a los alumnos sobre el carácter voluntario de la participación, garantizando en todo momento la confidencialidad de los datos, mediante su tratamiento global.

La aplicación fue desarrollada por dos investigadores entrenados tanto para explicar los objetivos al profesorado como para dar las instrucciones adecuadas al alumnado. Los cuestionarios se pasaron de forma colectiva en las distintas clases de Educación Primaria. El orden en el que se administraron las pruebas fue el siguiente: En una primera sesión, se aplicó el Inventario Infantil de Estresores Cotidianos (IIEC), el Inventario de Estrategias situacionales de afrontamiento en niños y niñas (ESAN) y el Registro de la profesión familiar. En una segunda sesión, se aplicó el Sistema de evaluación de la conducta en niños y adolescentes (BASC).

\section{Análisis de datos}

Con respecto al análisis de los datos, se procedió a examinar las relaciones, mediante análisis de correlación, que se establecen entre las medidas de afrontamiento infantil del inventario ESAN (situación de problema con los padres, ir al médico, problema con las notas y problema con los iguales) con otros instrumentos y constructos 
que ya han sido validados y cuyo uso está consolidado en distintas trayectorias de investigaciones y de estudios. Los análisis estadísticos han sido realizados con el paquete estadístico informatizado SPSS 15.00.

\section{RESULTADOS}

En cuanto a la situación de problema con los padres las asociaciones significativas de los factores del ESAN con dichas variables son las siguientes: a) La estrategia solución activa muestra correlaciones positivas con el ajuste personal de la BASC y correlación negativa con el desajuste escolar también de la BASC; b) La estrategia afrontamiento improductivo presenta una correlación positiva significativa con la variable desajuste escolar de la BASC; c) La estrategia evitación correlaciona positivamente con desajuste escolar, desajuste clínico e índice de síntomas emocionales de la BASC; y d) La estrategia emoción muestra correlaciones positivas significativas con la variable desajuste clínico de la BASC (Tabla 1).

Tabla 1. Coeficientes de correlación de Pearson del ESAN (en la situación problema con los padres) con el estrés cotidiano y las cuatro escalas globales del BASC

\begin{tabular}{|c|c|c|c|c|c|}
\hline Factores del ESAN & $\begin{array}{c}\text { Estrés } \\
\text { cotidiano }\end{array}$ & $\begin{array}{c}\text { Desajuste } \\
\text { Escolar }\end{array}$ & $\begin{array}{l}\text { Desajuste } \\
\text { Clínico }\end{array}$ & $\begin{array}{c}\text { Adaptación } \\
\text { Personal }\end{array}$ & $\begin{array}{c}\text { Síntomas } \\
\text { Emocionales }\end{array}$ \\
\hline Solución activa & .13 & $-.17 * *$ & .06 & $.17 * *$ & .03 \\
\hline A. improductivo & -.06 & $.11^{*}$ & -.01 & .06 & -.05 \\
\hline Evitación & .10 & $.14 * *$ & $.16^{* * *}$ & -.08 & $.11^{*}$ \\
\hline Emoción & .15 & -.08 & $.13 * *$ & .08 & .08 \\
\hline
\end{tabular}

Tabla 2. Coeficientes de correlación de Pearson del ESAN (en la situación ir al médico) con el estrés cotidiano y las cuatro escalas globales del BASC

\begin{tabular}{lccccc}
\hline Factores del ESAN & $\begin{array}{c}\text { Estrés } \\
\text { cotidiano }\end{array}$ & $\begin{array}{c}\text { Desajuste } \\
\text { Escolar }\end{array}$ & $\begin{array}{c}\text { Desajuste } \\
\text { Clínico }\end{array}$ & $\begin{array}{c}\text { Adaptación } \\
\text { Personal }\end{array}$ & $\begin{array}{c}\text { Síntomas } \\
\text { Emocionales }\end{array}$ \\
\hline Solución activa & -.04 & $-.09 *$ & -.02 & $.17 * *$ & -.08 \\
A. improductivo & $.24 *$ & $.24 * *$ & $.28 * *$ &. $.19 * *$ & $.27 * *$ \\
Evitación & -.08 & .02 & .09 & .00 & .06 \\
Emoción & .10 & .03 & $.19 * *$ & .04 & $.15 *$ \\
\hline Nota. A. improductivo $=$ afrontamiento improductivo; $*<<0.05 ; * p<0.01$ & &
\end{tabular}

En la situación de ir al médico aparecen los siguientes resultados: a) La estrategia solución activa muestra correlaciones positivas con el ajuste personal de la BASC. Demuestra correlación negativa significativa con desajuste escolar de la BASC; b) La estrategia afrontamiento improductivo muestra correlaciones positivas con el estrés cotidiano autopercibido (IIEC), con el desajuste escolar, desajuste clínico y síntomas emocionales de la BASC y muestra correlación negativa con la puntuación del ajuste personal de la BASC; c) La estrategia evitación no exhibe correlaciones positivas ni negativas significativas; y d) La estrategia emoción muestra correlaciones positivas con las variables desajuste clínico y síntomas emocionales de la BASC (Tabla 2). 
Con respecto a la situación de problema con las notas los resultados son las siguientes: a) La estrategia solución activa muestra correlaciones positivas con la variable ajuste personal de la BASC y muestra correlación negativa con la puntuación del desajuste escolar de la BASC; b) La estrategia afrontamiento improductivo muestra correlaciones positivas con el estrés cotidiano autopercibido (IIEC) así como con las puntuaciones de las variables desajuste escolar, desajuste clínico y síntomas emocionales de la BASC, mientras que muestra correlaciones negativas con la puntuación del ajuste personal de la BASC; c) La estrategia evitación exhibe correlaciones positivas con el estrés cotidiano autopercibido (IIEC), con la puntuaciones desajuste escolar, del desajuste clínico y síntomas emocionales de la BASC, mientras que muestra correlaciones negativas con la variable ajuste personal de la BASC; y d) La estrategia emoción no muestra correlaciones positivas en esta situación (Tabla 3).

Tabla 3. Coeficientes de correlación de Pearson del ESAN (en la situación problema con las notas) con el estrés cotidiano y las cuatro escalas globales del BASC

\begin{tabular}{lccccc}
\hline Factores del ESAN & $\begin{array}{c}\text { Estrés } \\
\text { cotidiano }\end{array}$ & $\begin{array}{c}\text { Desajuste } \\
\text { Escolar }\end{array}$ & $\begin{array}{c}\text { Desajuste } \\
\text { Clínico }\end{array}$ & $\begin{array}{c}\text { Adaptación } \\
\text { Personal }\end{array}$ & $\begin{array}{c}\text { Síntomas } \\
\text { Emocionales }\end{array}$ \\
\hline Solución activa & -.02 & $-.11^{*}$ & .09 & $.15^{*}$ & -.00 \\
A. improductivo & $.30^{* *}$ & $.36^{* *}$ & $.27 * *$ & $.23^{* *}$ & $.29 * *$ \\
Evitación & $.26^{* *}$ & $.36^{* *}$ & $.17 * *$ & $-.26^{* *}$ & $.24 * *$ \\
Emoción & -.06 & -.03 & .09 & .03 & .04 \\
\hline Nota. A. improductivo $=$ afrontamiento improductivo; $* p<0.05 ; * *<0.01$ & &
\end{tabular}

Tabla 4. Coeficientes de correlación de Pearson del ESAN (en la situación problema con los iguales) con el estrés cotidiano y las cuatro escalas globales del BASC

\begin{tabular}{lccccc}
\hline Factores del ESAN & $\begin{array}{c}\text { Estrés } \\
\text { cotidiano }\end{array}$ & $\begin{array}{c}\text { Desajuste } \\
\text { Escolar }\end{array}$ & $\begin{array}{c}\text { Desajuste } \\
\text { Clínico }\end{array}$ & $\begin{array}{c}\text { Adaptación } \\
\text { Personal }\end{array}$ & $\begin{array}{c}\text { Síntomas } \\
\text { Emocionales }\end{array}$ \\
\hline Solución activa & $-.19^{*}$ & $-.21^{* *}$ & .07 & $.20^{* *}$ & -.03 \\
A. improductivo & .13 & $.29^{* *}$ & $.17^{* *}$ & $-.17^{* *}$ & $.17^{* *}$ \\
Evitación & .08 & $.18^{* *}$ & $.10^{*}$ & -.04 & .09 \\
Emoción & -.12 & $-.12^{* *}$ & .00 & $.15^{* *}$ & -.06 \\
\hline Nota. A. improductivo $=$ afrontamiento improductivo; $* p<0.05 ; * *<0.01$ & &
\end{tabular}

Nota. A. improductivo $=$ afrontamiento improductivo; $* p<0.05 ; * * p<0.01$

En cuanto a la situación de problema con los iguales las correlaciones de los factores del ESAN con dichas variables son las siguientes: a) la estrategia solución activa muestra correlaciones positivas con la puntuación de adaptación personal y demuestra correlaciones negativas con el estrés cotidiano autopercibido y con desajuste escolar; b) la estrategia afrontamiento improductivo muestra correlaciones positivas con desajuste escolar, desajuste clínico y síntomas emocionales, mientras que muestra correlaciones negativas con adaptación personal; c) la estrategia evitación demuestra correlaciones positivas con las puntuaciones del desajuste escolar y desajuste clínico; y d) la estrategia emoción exhibe correlaciones positivas con adaptación personal y demuestra correlaciones negativas con el desajuste escolar (ver tabla 4). 


\section{DISCUSION}

En el enfoque situacional que se practica en este estudio tiene sentido comentar las correlaciones entre ESAN y IIEC a partir de las cuatro situaciones empleadas ya que las distintas situaciones o problemas llevan al niño o niña a emplear distintas estrategias de afrontamiento dependiendo de las características de la situación (Cano, García y Rodríguez, 2004; Cassaretto, Chau, Oblitas y Valdez, 2003). En cuanto a las asociaciones entre la puntuación del IIEC y los factores del ESAN aparece que, en la primera situación (padres), el IIEC no correlaciona significativamente con ningún factor del ESAN. En la segunda (médico) y tercera situación (notas), el estrés (IIEC) correlaciona con el afrontamiento improductivo y además, con el factor evitación, en la tercera situación, mientras que en la cuarta (iguales) se asocia inversamente con solución de problemas. Se deduce que el estrés no guarda relación con un patrón determinado de afrontamiento en la situación estresante de afrontar problemas entre los padres. Se asocia en cambio a afrontamiento improductivo y evitación en situaciones que no se prestan a una acción directa por parte del niño o niña mientras que correlaciona con bajas habilidades de solución de problemas en los conflictos con iguales. Otros estudios encuentran relación negativa entre el estrés vivido y el empleo de soluciones constructivas ante los problemas sociales en la infancia (Causey y Dubow, 1992; Pelegrín y Garcés de Los Fayos, 2008).

En cuanto a la asociación del estrés con factores de afrontamiento improductivo y de evitación, las relaciones entre evitación y estrés cotidiano están presentes en la literatura (Causey y Dubow, 1992; Kirchner, Forns y Amador, 2004). En cuanto al afrontamiento cabe destacar que el estrés cotidiano infantil, produce correlaciones, de acuerdo con la literatura, positivas con factores de afrontamiento improductivo y disfuncional y negativas con estrategias de afrontamiento productivo y funcional. Al mismo tiempo, se encuentra, en niños, que el empleo de estrategias de afrontamiento improductivo se asocia a mayor vulnerabilidad al estrés cotidiano (Compas et al., 1993; Dumont y Provost, 1999; Sandín, 2003; Seiffge-Krenke, 2000). La aplicación práctica de este resultado es evidente, ya que el enseñar estrategias de afrontamiento será más fácil en niños y niñas no estresados, y en aquellos que presenten estrés en sus vidas les ayudará a disminuir el estrés. Supone pues una vía de enseñanza de estrategias de salud mental en las poblaciones escolares en edades en las que puede haber una amplia repercusión en sus vidas.

En cuanto a las correlaciones de los factores del BASC con el ESAN se demuestran 35 correlaciones significativas de las variables del BASC con los factores del ESAN a través de patrones que se repiten a veces en las distintas situaciones. Comenzando por el desajuste clínico puede observarse que correlaciona con el afrontamiento improductivo en tres situaciones excepto la primera (familia) mientras que 
correlaciona con el factor evitación del ESAN también en tres situaciones excepto la segunda (médico). También correlaciona desajuste clínico con emoción del ESAN en dos situaciones (familia y médico). Estos resultados aluden a que, el alumnado vulnerable a sufrir desajustes clínicos, tiende a emplear afrontamiento improductivo en situaciones de estrés relacionado con actos médicos, malas notas y problemas con los iguales. También puede emplear evitación al enfrentarse a problemas interpersonales relacionados con la familia, con las notas y con los iguales. El desajuste clínico es un síndrome que conlleva dificultades y problemas en el entorno del niño o niña que lo sufre, por no saber o poder afrontar hábilmente esas dificultades (Grant et al., 2006).

Se comenta ahora el caso del desajuste escolar, el cual correlaciona con afrontamiento improductivo en las cuatro situaciones y con evitación en tres de las situaciones, excepto la segunda (médico). Estos resultados aluden a que no tener un afrontamiento hábil de los problemas familiares, escolares, médicos o entre iguales, tiene un peso en sufrir desajuste escolar, al igual que encuentran otros estudios (Griffith et al., 2000). Además cuanta más puntuación se obtenga en desajuste escolar menor puntuación se espera en el uso de un afrontamiento basado en la emoción, en la situación de problemas con iguales. Este resultado dibuja un papel positivo, en la resolución de problemas con iguales, para el afrontamiento basado en la emoción, o dicho de otra manera, la emoción es productiva en el afrontamiento de los problemas con los iguales. Este resultado es novedoso ya que existen otros resultados no congruentes como los de Band y Weisz (1990), quienes encuentran peor ajuste psicológico cuando el afrontamiento está centrado en la estrategia emoción. Claro que estos autores no especifican el contexto de forma tan precisa como lo hace el ESAN. Por último, en las cuatro situaciones, la puntuación en desajuste escolar es inversa a la de solución de problemas demostrando que, para un niño o niña, en contextos escolares es muy valorado poseer estrategias de solución de problemas interpersonales e incompatible con ser evaluado con desajuste escolar (Demaray, Malecki, Davidson, Hodgson y Rebus, 2005).

Pasamos ahora a comentar las asociaciones demostradas por la variable de la BASC llamada sintomatología emocional, con los factores del ESAN. Es la variable de la BASC que demuestra menos asociaciones con el ESAN. De hecho, solo afrontamiento improductivo (en 3 situaciones), evitación (en dos) y emoción (en una) demuestran correlacionar. El afrontamiento improductivo no participa en la situación 1 (familia), evitación no correlaciona en la situación 2 (médico) y 4 (iguales) y emoción lo hace solo en la situación de ir al médico. Se deduce así que alumnado con alta puntuación en sintomatología emocional demuestra predisposición para emplear afrontamiento improductivo en problemas con actos médicos, con las notas y con los iguales, mientras que no en problemas familiares, en los que prefiere evitación que puede implicar cierta huida. Otros estudios que investigan estresores del ámbito escolar y de los iguales 
encuentran también que el mayor empleo de estrategias de tipo evitativo predice menos ajuste personal (González, Montoya, Casullo y Bernabéu, 2002; Griffith et al., 2000). También se asocia de forma directa la puntuación en trastorno emocional y el empleo de evitación en el problema con las notas. Puede decirse que el empleo de estrategias de afrontamiento improductivo se asocia, en la literatura, a menor adaptación socioemocional (Cabanach et al., 2010; Seiffge-Krenke y Stemmler, 2002). Por último cabe comentar el mayor empleo de emoción en los que sufren desajuste emocional en la situación de ir al médico. Otras investigaciones demuestran que en situación de afrontamiento de actos médicos los niños y niñas tienen en el manejo de la emoción el principal reto y si fallan en este manejo, las consecuencias se hacen presentes en síntomas emocionales (Donaldson, Prinstein, Danovsky y Spirito, 2000; GómezFraguela, Luengo, Romero, Villar y Sobral, 2006; Seiffge-Krenke, 2000).

Antes de pasar a comentar los resultados con la escala positiva del BASC, adaptación personal, cabe poner de relieve que, en términos generales, y en consonancia con los resultados obtenidos en estudios previos, el afrontamiento improductivo se relaciona directamente con problemas socioemocionales, escolares y peor ajuste personal (Dumont y Provost, 1999; Frydenberg y Lewis, 1996b; Frydenberg y Lewis, 2004; Seiffge-Krenke, 1993a; Seiffge-Krenke, 2000). Es evidente que las estrategias de afrontamiento llamadas improductivas o disfuncionales presentan en estos y otros datos una fuerte asociación con psicopatología e inadaptación, encontrándose al mismo tiempo una vía de prevención y disminución de riesgos en la infancia en la enseñanza de estrategias de afrontamiento productivas y funcionales ante los problemas familiares, escolares, de los iguales y en relación con actos médicos.

Finalmente se discuten los resultados obtenidos por asociaciones de la escala adaptación personal de la BASC y los factores del ESAN. Las correlaciones entre el empleo de la estrategia de afrontamiento solución activa y la escala de ajuste personal del BASC están presentes en las cuatro situaciones. Esta asociación está presente también en otros trabajos que muestran que el empleo de esa estrategia que puede ser considerada de afrontamiento productivo protege frente a la experiencia de estrés en diversas situaciones infantiles (González et al., 2002; Isakson y Jarvis, 1999).

El ESAN evalúa afrontamiento infantil ante estresores cotidianos manteniendo un enfoque ajustado a las características de la situación, que como vemos ha generado resultados diferentes en cada uno de los cuatro contextos evaluados. Este trabajo supone una primera aproximación a la demostración del carácter ajustado a situación del cuestionario ESAN, a través de correlaciones directas. Esta característica le confiere un valor distintivo al ESAN ya que otros instrumentos en el mercado se organizan en función de las estrategias (Kirchner et al., 2004) o los estilos de afrontamiento (Frydenberg y Lewis, 1996b) pero no específicamente por problemas o situaciones. Puede por tanto el ESAN presentar una utilidad especial para el clínico, el 
orientador, o el investigador en el campo del afrontamiento infantil. Finalmente la información aportada por los datos obtenidos en el presente estudio podría ser útil para el diseño de programas de intervención psicoeducativa para población escolar, dirigidos a promover el conocimiento y empleo de estrategias de afrontamiento eficaces y productivas ante distintos tipos de problemas frecuentes en la infancia y adolescencia.

En el futuro sería preciso seguir profundizando en el análisis del carácter ajustado a situación del cuestionario principalmente mediante analítica de AFC. También sería preciso desarrollar diseños longitudinales que permitan evaluar cómo se transforman las estrategias de afrontamiento desde la infancia hasta la adolescencia, ajustando este cambio a las distintas situaciones relevantes en esos dos momentos del ciclo vital. Estos dos enfoques y otros contribuirían a evaluar y profundizar en la aportación específica de un cuestionario de afrontamiento ajustado a las características de la situación.

Agradecimientos

Este trabajo ha recibido financiación del proyecto SEJ 2007-61447.

\section{REFERENCIAS}

Band, E. y Weisz, J.R. (1990). Developmental differences in primary and secondary control coping and adjusment to juvenil diabetes. Journal of Clinical Child Psychology, 19, 150-158.

Cabanach, R.G., Valle, A., Rodríguez, S., Piñeiro, I. y González, P. (2010). Las creencias motivacionales como factor protector del estrés en estudiantes universitarios. European Journal of Education and Psychology, 3(1), 75-87.

Cano, F.J., García, J. y Rodríguez, L. (2004). Contextual assessment of stress coping strategies. IV European Conference on Psychological assessment. EAPA 2004.

Casaretto, M., Chau, C., Oblitas, H. y Valdez, N. (2003). Estrés y afrontamiento en estudiantes de Psicología. Revista de Psicología de la Pontificia Universidad Católica del Perú. Perú: Fondo Editorial, volumen XXI.

Causey, D. y Dubow, E. (1992). Development of a self-report coping measure for elementary school children. Journal of Clinical child Psychology, 21, 47-59.

Compas, B.E., Malcarne, V.L. y Fondacaro, K.M. (1988). Coping with stressful events in older children and young adolescents. Journal of Consulting and Clinical Psychology, 56, 405-411.

Compas, B.E., Orosan, P.G. y Grant, K.E. (1993). Adolescent stress and coping: Implications for psychopathology during adolescence. Journal of Adolescence, 16, 331-349.

Demaray, M.K., Malecki, C.K., Davidson, L.M., Hodgson, K.K. y Rebus, P.J. (2005). The relationship between social support and student adjustment: A longitudinal analysis. Psychology in the Schools, 42(7), 691-706.

Donaldson, D., Prinstein, M. J., Danovsky, M. y Spirito, A. (2000). Patters of children`s coping with life stress: Implications for clinicians. American Journal of Orthopsychiatry, 70(3), 351-359.

Dumont, M. y Provost, M.A. (1999). Resilience in adolescents: Protective role of social support, coping strategies, self-esteem, and social activities on experience of stress and 
depression. Journal of Youth and Adolescence, 28, 343-363.

Frydenberg, E. y Lewis, R. (1996b). A replication study of the structure of the Adolescent Coping Scale: Multiple forms and applications of a self-report inventory in a counselling and research context. European Journal of Educational Psychological Assessment, 12(3), 224-235.

Frydenberg, E. y Lewis, R. (2004). Adolescents least able to cope: how do they respond to their stresses? British Journal of Guidance \& Counselling, 32(1), 25-37.

Gómez-Fraguela, J.A., Luengo, A., Romero, E. Villar, P. y Sobral, J. (2006). Estrategias de afrontamiento en el inicio de la adolescencia y su relación con el consumo de drogas y la conducta problemática. International Journal of Clinical and Health Psychology, 6(3), 581-597.

González, R., Montoya, I., Casullo, M.M., y Bernabéu, J. (2002). Relación entre estilos y estrategias de afrontamiento y bienestar psicológico en adolescentes. Psicothema, 14(2), 363-368.

González, J., Fernández, S., Pérez, E. y Santamaría, P. (2004). Adaptación española de sistema de evaluación de la conducta en niños y adolescentes: BASC. Madrid: TEA Ediciones.

Grant, K.E., Compas, B.E., Thurm, A.E., McMahon, S.D., Gipson, P.Y., Campbell, A.J., Krochock, K. y Westerholm, R.I. (2006). Stressors and child and adolescent psychopathology: Evidence of moderating and mediating effects. Clinical Psychology Review, 26, 257-283.

Griffith, M.A., Dubow, E. e Ippolito, M.F. (2000). Developmental and cross-situational differences in adolescents`coping strategies. Journal of Youth and Adolescence, 29(2), 183-204.

Halama, P. y Bakosova, K. (2009). Meaning in life as a moderator of the relationship between perceived stress and coping. Studia Psychologica, 51(2-3), 143-148.

Isakson, K. y Jarvis, P. (1999). The adjustment of adolescents during the transition into high school: a short-term longitudinal study. Journal of Youth and Adolescence, 28(1), 1-26.

Keane, S.P. y Calkins, S.D. (2004). Predicting kindergarten peer social status from toddler and preschool problem behavior. Journal of Abnormal Child Psychology, 32, 409-423.

Kirchner, T., Forns, M. y Amador, J.A. (2004). Especificidad en el uso de estrategias de afrontamiento. VII European Conference on Psychological assessment. EAPA 2004.

Lapointe, V. y Marcotte, D. (2000). Gender typed characteristics and coping strategies of depressed adolescents. European Review of Applied Psychology, 50(4), 451-460.

Morales, F. M., Trianes, M.V., Miranda, J., Fernández, F.J. y Escobar, M. (2008). Assessment of coping strategies in children: development and preliminary analysis of the psychometric properties of a new instrument. Enviado para publicar en European Journal of Psychological Assessment.

Pelegrín, A. y Garcés de Los Fayos, E.J. (2008). Variables contextuales y personales que inciden en el comportamiento violento del niño. European Journal of Education and Psychology, 1(1), 5-20.

Reynolds, C. y Kamphaus, R.W. (1992). Behavior assessment system for children-BASC. Circle Pines: American Guidance Service.

Sandín, B. (2003). El estrés: un análisis basado en el papel de los factores sociales. International Journal of Clinical and Health Psychology, 3, 141-157.

Seiffge-Krenke, I. (1993a). Introduction to special issue on stress and coping. Journal of Adolescence, 16, 227-233.

Seiffge-Krenke, I. (2000). Causal links between stressful events, coping style and adolescent symptomatology. Journal of Adolescence, 23, 675-691.

Seiffge-Krenke, I. y Stemmler, M. (2002). Factors contributing to gender differences in 
depressive symptoms: A test of three developmental models. Journal of Youth and Adolescence, 31, 405-417.

Trianes, M.V., Blanca, M.J., Fernández, F.J., Escobar, M., Maldonado, E. y Muñoz, A. (2009). Evaluación del estrés infantil: Inventario Infantil de Estresores Cotidianos (IIEC). Psicothema, 21(4), 598-603.

Vendette, K. y Marcotte, D. (2000). The moderator`s role of social support on the relation between coping and depression during adolescence. Science et Comportement, 28(2), 129-144.

Recibido: 5 de enero de 2010

Recepción Modificaciones: 19 de abril de 2010

Aceptado: 22 de abril de 2010 